\section{Resistance in multiple myeloma}

\begin{abstract}
A key feature of multiple myeloma is the buildup of
\end{abstract} immunoglobulin-secreting plasma cells in the bone marrow. To accommodate the synthesis of secretory immunoglobulin, the endoplasmic reticulum (ER) of these cells expands, and it is thought that proteasome inhibitors hinder ER function by blocking proteasome-assisted, ER-associated degradation. Now, Rodger Tiedemann and colleagues report a new mechanism of intrinsic proteasome inhibitor resistance in multiple myeloma (Cancer Cell 24, 289-304, 2013). In reanalyzing genome-wide small interfering RNA (siRNA) screening data, the authors noticed that the gene IRE1 was required for proteasome inhibitor-induced cell death in a multiple myeloma cell line. Silencing of IRE1 led to proteasome inhibitor resistance in six other multiple myeloma cell lines. Ire1 signaling is mediated via the transcription factor $\mathrm{Xbp} 1$, and analysis of gene expression in tumor samples pretreated with proteasome inhibitor showed that all Xbp1 target genes had lower expression in multiple myeloma tumors that subsequently appeared refractory to proteasome inhibitor treatment. The authors purified plasma cell progenitor populations present in multiple myeloma tumors and found that such cells were negative for Xbp1s. The authors conclude that such Xbp1s-negative plasma cell progenitors are intrinsically insensitive to proteasome inhibitor, which may account for failures in treating multiple myeloma with proteasome inhibitor.

\section{Embryonic epigenetic chimerism}

To gain insight into how epigenetic aberrations arise during genomic reprogramming in mammalian preimplantation embryos, Daniel Messerschmidt, William Burkholder and colleagues developed an assay to measure DNA methylation in single cells (Science 341, 1110-1112, 2013). They analyzed DNA methylation at six imprinted loci after genetically manipulating Trim28, which encodes a DNMT1-binding protein and is known to be required for the maintenance of differential methylation at imprinted loci in preimplantation embryos. They examined blastomeres from eight-cell embryos after maternal Trim 28 knockout and observed variable aberrant methylation patterns indicative of embryos that consisted of mixtures of normally and aberrantly imprinted cells. The authors further defined the temporal requirement for TRIM28 by transferring control or maternal Trim28-null pronuclei into enucleated control or Trim28-null zygotes. They found that removal of maternal TRIM28 reduced zygote survival, and supply of maternal TRIM28 increased zygote survival, showing that maternal TRIM28 is required after fertilization. These authors previously showed that loss of maternal TRIM28 causes phenotypic variability and embryonic lethality. This new work demonstrates that loss of maternal TRIM28 creates epigenetic chimeras and may explain the phenotypic variability of the embryos that result from loss of maternal TRIM28.

\section{Hedgehog agonist for Down syndrome}

One characteristic of Down syndrome and the Ts65Dn mouse is a smaller and hypocellular cerebellum. The canonical Sonic Hedgehog (Shh) pathway is important for cerebellar development, and, now, Roger Reeves and colleagues show that a single treatment of Tn65Dn mice with a Hedgehog agonist leads to normal cerebellum in adults (Sci. Transl. Med. 5, 201ra120, 2013). SAG 1.1 (SAG), a known Shh pathway agonist, binds and activates

Written by Pamela Feliciano, Emily Niemitz \& Kyle Vogan
Smoothened, thereby activating the canonical Shh pathway. This molecule crosses the blood-brain barrier and can activate the proliferation of granule cell precursors in newborn mice. The authors administered SAG to newborn Ts65Dn mice and found that, at 15 weeks of age, SAG-injected mice had the same density of granule cells as control mice. Interestingly, despite the obvious morphological normalization of SAG-injected Ts65Dn mice, long-term depression originating in cerebellar Purkinje cells was not normalized. Nevertheless, behavioral tests showed that SAG-injected Ts65Dn mice had significant improvements in hippocampal tasks involving learning and memory but not in prefrontal tasks. Although the results suggest that SAG may have a therapeutic role in Down syndrome, further work needs to proceed with caution, as activating the Shh pathway in newborns may have deleterious effects, given the known role of Shh stimulation in medulloblastoma.

$P F$

\section{Epileptic encephalopathy genes}

Several genes have been implicated in early-onset epileptic encephalopathies, but in most cases the underlying causes remain unknown. Two independent studies have now applied exome sequencing to identify new causative mutations for these rare disorders. Lina Basel-Vanagaite, Guntram Borck and colleagues (Am. J. Hum. Genet. 93, 524-529, 2013) analyzed two unrelated individuals with early-onset epileptic encephalopathy and overlapping clinical features and identified biallelic truncating mutations in SZT2 in both subjects. Notably, mice with mutations in Szt2 are susceptible to induced seizures (Genes Brain Behav. 8, 568-576, 2009), mirroring the human phenotype. Separately, Hirotomo Saitsu and colleagues (Am. J. Hum. Genet. 93, 496-505, 2013) identified de novo mutations in GNAO1 in four individuals with epileptic encephalopathy characterized by severe intellectual disability and delays in motor development. GNAO1 encodes a heterotrimeric $\mathrm{G}$ protein $\alpha$ subunit that is known to transduce signals from adrenergic receptors and modulate the activity of calcium channels. Two of the four individuals with GNAO1 mutations also exhibited involuntary movements, and three were diagnosed with Ohtahara syndrome, highlighting the importance of $\mathrm{G}$ protein signaling for proper neural development.

$K V$

\section{Neocortex enhancers}

Gill Bejerano and colleagues (PLoS Genet. 9, e1003728, 2013) have generated a map of active enhancers in the region of the developing mouse brain that gives rise to the neocortex. The authors dissected the dorsal cerebral wall at embryonic day (E) 14.5 and performed chromatin immunoprecipitation and sequencing (ChIP-seq) with an antibody against p300, a marker of active enhancers. They identified 6,629 sites enriched for $p 300$ binding, $95 \%$ of which show evolutionary conservation in humans and $20 \%$ of which overlap with a previously published map of p300 binding sites from E11.5 mouse forebrain (Cell 152, 895$908,2013)$. They subsequently tested ten of the newly discovered candidate enhancer elements in transgenic reporter assays and found that eight of them could drive expression reproducibly in the developing neocortex. The predicted enhancers are enriched near genes known to be important for brain development, including several key transcription factors and signaling molecules. They are also enriched for transcription factor binding motifs for known regulators of neocortex development. The authors also identified several genes showing a very high density of $\mathrm{p} 300$ peaks. This new enhancer map provides a framework for studying the regulatory networks driving neocortical development and evolution. KV 